\title{
Superfast Liquid Transfer Strategy Through Sliding on a Liquid Membrane Inspired from Scorpion Setae
}

\author{
Daobing Chen, Shichao Niu,* Junqiu Zhang, Zhengzhi Mu, Huwei Chen, \\ Depuan Zhang, Zhongwen Yao, Zhiwu Han,* and Luquan Ren
}

\begin{abstract}
Although diversified biological structures have evolved fog collection abilities, the typical speeds of the condensed water droplets on these surfaces are too slow to have practical utility. The main challenge focuses on the elimination of the interfacial hydrodynamic resistance without external energy support. Here, an unusual strategy for superfast self-support transfer condensed droplets is supported by sliding on seta of desert scorpion. It can be rapidly wetted by the fog droplets owing to its conical shape with linear gradient channels. A loss of interfacial resistance by this hydrodynamically lubricating water membrane could significantly accelerate the movement of the droplets, thus making its velocity increasing by one order of magnitude, or even more. Inspired by this novel strategy, the novel bioinspired materials are fabricated with the similar gradient channel structures and droplet transportation mode, which can make the condensed droplets spontaneously slide on the low-friction liquid membrane. The fundamental understanding of superfast fog capture and the sliding dynamics of condensed droplets in this system could inspire to develop novel materials or various systems to transfer liquid fast and efficiently without external energy support.
\end{abstract}

Many biological surfaces in both the plants and animals possess unique structural features at the micro- and nanoscale. They are able to control their interaction with water and hence wettability. ${ }^{[1-8]}$ Many species living in arid deserts are extremely drought-tolerant. In addition to the adaptive characteristics that minimize water loss, some species appear to use fog as an additional water supply by the unique structural features on their surfaces, such as gradient grooves on cactus spine, ${ }^{[4,5,9]}$ sand moss wan, ${ }^{[3]}$ bumps on the back of desert beetles, ${ }^{[7,10,11]}$ and periodic spindle knots and joints on spider silk. ${ }^{[12]}$ However, the typical velocity of the droplets on these surfaces is so slow that it is hard to have practical utility.

Here, we report that the setae on Parabuthus transvaalicus (P. transvaalicus), one kind of desert scorpion, can also capture water from the fog. However, the efficiency of its transporting condensed droplets on its setae surface is much

Dr. D. B. Chen, Dr. S. C. Niu, Dr. J. O. Zhang, Dr. Z. Z. Mu,

Prof. Z. W. Yao, Prof. Z. W. Han, Prof. L. Q. Ren

Key Laboratory of Bionic Engineering

Ministry of Education

Jilin University

Changchun 130022, P. R. China

E-mail: niushichao@jlu.edu.cn; zwhan@jlu.edu.cn

Prof. H. W. Chen, D. Y. Zhang

School of Mechanical Engineering and Automation

Beihang University

Beijing 100191, China

Dr. J. Q. Zhang

Department of Mechanical Engineering

Columbia University

NY 10027, USA

Dr. Z. Z. $\mathrm{Mu}$

Department of Chemical Engineering

University of Michigan

Ann Arbor, MI 48109, USA

Prof. Z. W. Yao

Department of Mechanical and Materials Engineering

Queen's University

Kingston K7L 3N6, Canada

The ORCID identification number(s) for the author(s) of this article can be found under https://doi.org/10.1002/admi.201800802.

DOI: $10.1002 / \mathrm{admi} .201800802$ higher than the cases previously reported. The underlying mechanism is fully revealed in this work. In fact, a thin liquid membrane can rapidly form on the setae surface, and then it could significantly speed up the velocity of droplets in the mode of sliding on the liquid membrane with extremely low frictional resistance. This sliding mode with low resistance on liquid membrane can greatly improve the liquid transport efficiency.

The optical image of P. transvaalicus illustrates the appearance characteristics of this representative desert scorpion (Figure 1a). It belongs to Arthropoda, Arachricla, Scorpionida, and Buthidae and mainly lives in the extremely arid desert areas of southern Africa. There are thousands of setae distributed on the pincers and other parts of this scorpion (Figure $1 \mathrm{~b}$ and Figure S1, Supporting Information). To investigate structures of the setae in detail, a scanning electron microscope (SEM) was adopted to observe an individual seta (Figure 1c-h). It can be seen from Figure 1c that the setae are mostly perpendicular to the pincer body surface and point radially outward. Adjacent setae are not staggered with each other. They all grow as a vertical needle-like array on the scorpion surface with variable distances ranging from $\approx 100$ to $500 \mu \mathrm{m}$. The structural parameters of the setae are $800-1300 \mu \mathrm{m}$ in length and $10-25 \mu \mathrm{m}$ in diameter. Figure $1 \mathrm{~d}$ shows that the seta is composed of a short seta shaft and a relatively large socket (Figure $1 \mathrm{~h}$ and Figure S2, 

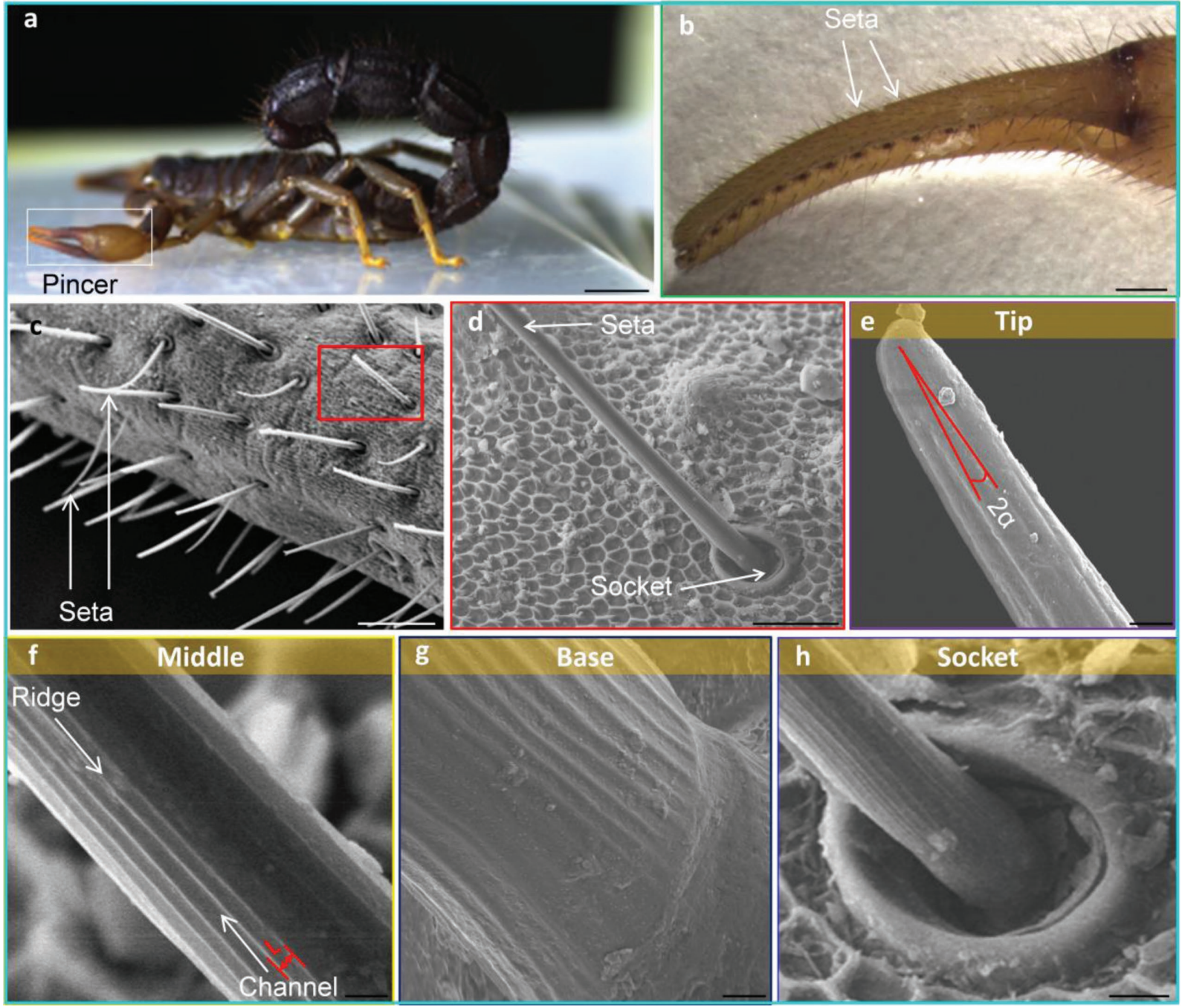

Figure 1. Location, appearance, and surface structure of the scorpion setae. a) Optical image of the desert scorpion $P$. transvaalicus. b) Pincer of this desert scorpion is covered with large numbers of setae. c) SEM image of the seta located on the pincers of the desert scorpion. d) SEM image of a single seta. e-h) SEM images show the details of the surface of a single seta in four different regions. e) Magnified image of the tip with an apex angle $(2 \alpha)$. Magnified images of the e) tip, f) middle, and g) base show the gradient channels. h) Magnified image of the socket connect with the seta shaft. The number of the channels $(\mathrm{N})$ increases from e) the tip to $\mathrm{g}$ ) the base (tip, 3; middle, 10; base, 17). The width of the channels decreases from the base to the tip. $\alpha$ : half apex angle; $L$ : width of channel. Scale bars, a) $1 \mathrm{~cm}$, b) $500 \mu \mathrm{m}$, c) $100 \mu \mathrm{m}$, d) $50 \mu \mathrm{m}, \mathrm{e}-\mathrm{g}) 2 \mu \mathrm{m}$, and h) $10 \mu \mathrm{m}$.

Supporting Information). Figure 1e-g shows the magnified images of different positions of a seta shaft. From Figure 1e, it can be seen that the seta has a small conical shape with an apex angle $(2 \alpha)$ of $3^{\circ}-5^{\circ}$. Figure $1 \mathrm{f}$ reveals the parallel channels and ridges with exquisite linear alternating distribution. The channels present a gradient in width (L) along the seta shaft. Its width changes from an average of $\approx 2 \mu \mathrm{m}$ near the base to an average of $\approx 0.5 \mu \mathrm{m}$ near the tip (Figure S3a, Supporting Information). At the same time, the number $(\mathrm{N})$ of channels also gradually decreases from the base to the tip, ranging from less than $\approx 4$ channels to more than $\approx 50$ channels (Figure 1e-g, Figure S3c,d, Supporting Information). Compared to cactus spine and sand moss awn, the surface structures of a single seta on desert scorpion examined here have no hierarchical or multi-level grooves. The channels on the seta surface of this desert scorpion are elaborately arranged and lined up from the tip to the base.
The ability of the scorpion seta to capture water from fog is investigated by using a saturated fog flow with a velocity of $\approx 20$ to $30 \mathrm{~cm} \mathrm{~s}^{-1}$ (see Experimental Section). The movement of condensed water droplets is recorded exhaustively by a highspeed camera, with microscopic magnification lens in a loop recording mode. As setae are distributed on the desert scorpion in all directions, the directional movement behaviors of the water droplets on different seta under different angles $\left(0^{\circ}, 45^{\circ}\right.$, and $90^{\circ}$ ) are determined (Figure 2). For each angle, all the water droplets can ultimately climb along the seta shaft from the tip side to the base side, even when the seta shaft vertically pointed to the ground (Figure 2b-e). However, the efficiency is not consistent throughout the entire water droplet transport process. In fact, it can be clearly divided into two stages. For the initial stage as shown in Figure 2a-c, the volume of the water droplets increases constantly until their radius reaches about $50 \pm 5 \mu \mathrm{m}$, 


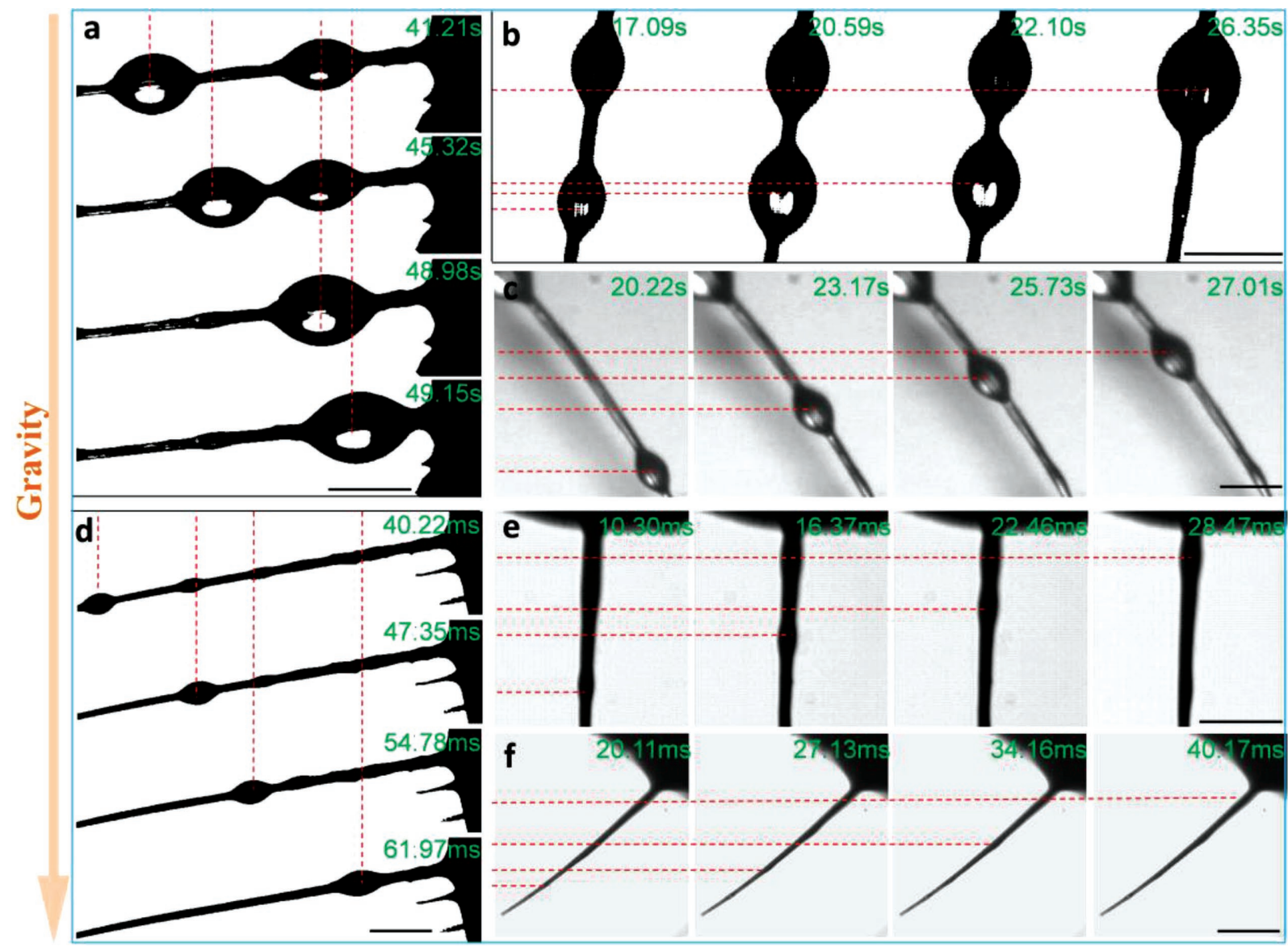

Figure 2. In situ optical microscope observation of fog capture process of the setae located at various angles. a-f) Setae are arranged in different tilt angle: $0^{\circ}, 45^{\circ}$, and $90^{\circ}$ relative to the horizontal direction, respectively. b,e) The deposited water droplets (red arrows) are moving along the tip to the base of the seta, even the seta vertically oriented with the tip pointing down. The size and the moving velocity of these droplets are different. The droplets on panels $(\mathrm{a}-\mathrm{C})$ are big and moving slow, but the droplets are small and moving fast on panels (d-f). Scale bar $=100 \mu \mathrm{m}$.

then they start to transport toward the root position. The whole process is completed in a relatively short time. In contrast, at the second stage, the threshold at which the droplet begins to move becomes lower $(10 \mu \mathrm{m}$ in Figure $2 \mathrm{~d}-\mathrm{f})$. But, the water transport velocity is unexpectedly higher. It can be seen from Figure S4, Supporting Information, by comparing the fog collecting efficiency of the legs of scorpions with or without aetae, that there are one water drop produced mean interval of $50 \mathrm{~s}$. The fog-water harvesting volume per minute is about $0.1 \mathrm{~mL}$. In fact, there are two different water transport modes appeared at the two stages. Obviously, the water transport efficiency of the second stage is significantly better than that of the first one, which is also much higher than the cases previously reported. ${ }^{[2,3,5,6,11,12]}$ All the above results indicated that the superfast movement of water droplets at the second stage on the setae might be driven by other greater forces or under low resistance conditions.

To reveal the fog capture process of the setae and determine the two modes of water transport, a single vertical seta is investigated (Figure 3 and Movie S1, Supporting Information). Initially, the fog flow is captured by the dry seta and quickly condensed into small water droplets on the seta shaft. Numerous tiny droplets coalesce with adjacent smaller ones, forming seven individual droplets that are marked 1-7 (Figure 3a). As the deposition of the fog continues, the droplets become much bigger and then move toward the base of the seta shaft. Coalescence will continue between different droplets, thereby forming larger droplets. For the sake of intuitive description of the droplets movement patterns, we use the average speed to evaluate their movements due to it is difficult to measure the instantaneous velocity of the droplets. The average velocity of the droplet $1\left(v_{1}\right)$ moving to the droplet 2 is $\approx 7 \mu \mathrm{m} \mathrm{s}^{-1}$. After their coalescence, the average velocity of the new droplet $1+2\left(v_{2}\right)$ is $\approx 9 \mu \mathrm{m} \mathrm{s}^{-1}$. Once the droplet $1+2$ merged with the droplet 3 and formed a new one $1+2+3$, the average velocity of this new droplet $1+2+3$ $\left(v_{3}\right)$ changes to $\approx 11.7 \mathrm{~mm} \mathrm{~s}^{-1}$. Soon after, the latest droplet $1+2+3+4+5$ formed via further coalescence and its average velocity $\left(v_{4}\right)$ is $\approx 1.2 \mathrm{~mm} \mathrm{~s}^{-1}$. All the process of the droplet 1 moving and continuously coalescing with other droplets (droplet 2-7) can be defined as the first mode (from 0 to $17.25 \mathrm{~s}$ ). In this mode, the droplets move rather slowly with an average velocity, which is only about $30.93 \mu \mathrm{m} \mathrm{s}^{-1}$ (Figure 3b). In fact, once the biggest water droplet reaches to the root position of the seta, there will be no any other big droplets continuously formed at all other parts as shown in Figure 3a. Therefore, the initial stage completes and the next stage starts. 

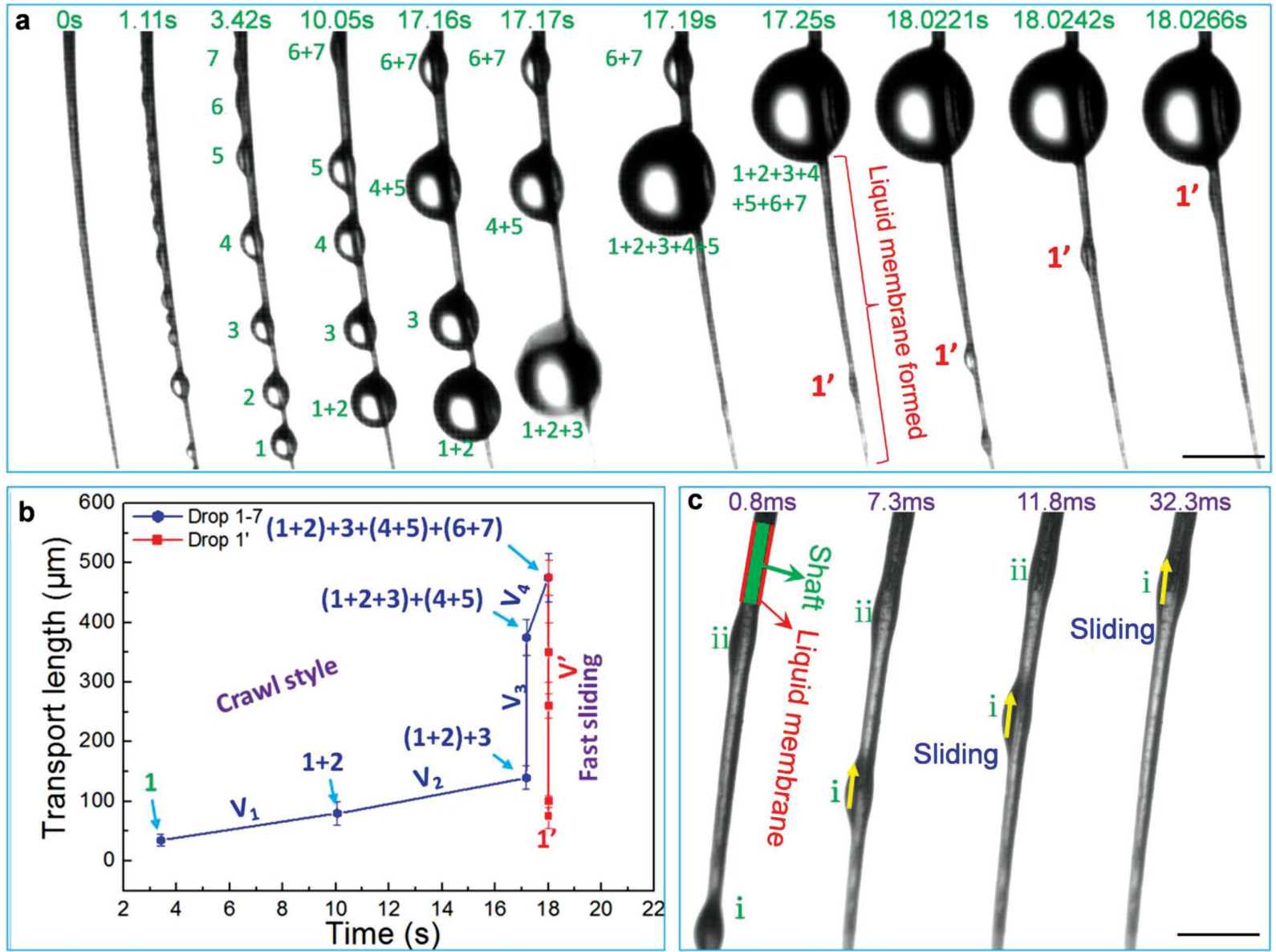

Figure 3. In situ optical microscope observation of the detailed water capture process on a single vertical seta to determine the different transport mode. a) Water transport mode changes from the crawl mode to the superfast sliding mode on the liquid membrane as the sequences of time. Initially, the tiny water droplets are deposited concomitantly on the seta shaft. As the deposition processing, the tiny droplets are identified with seven separate ones marked 1, 2, 3, 4, 5, 6, and 7, respectively. Then, the droplet 1 coalesces with droplet 2 . Droplet 4 coalesces with droplet 5 . Droplet 6 coalesces with droplet 7. So, they form three larger droplets $1+2,4+5$, and $6+7$, respectively. Larger droplet $1+2$ keeps moving toward the seta base and coalesces with droplet 3 , forming droplet $1+2+3$. Droplet $1+2+3$ keeps moving and coalesces with droplet $4+5$, forming larger droplet $1+2+3+4+5$. Finally, larger droplet $1+2+3+4+5$ moves to droplet $6+7$ and coalesces, forming the largest droplet $1+2+3+4+5+6+7$. The crawl mode of droplets is ended at this time. The entire surface of the seta is wetted and a liquid membrane is formed after the droplets passing through. The superfast sliding mode starts. A new tiny droplet $\mathrm{I}^{\prime}$ forms on the wetted seta shaft and fast slides on surface of the seta with the volume decreasing as the time sequence. b) Moving distances, corresponds to time, of the droplets (droplets 1-7) and subsequent droplet (droplet $7^{\prime}$ ). The velocity of the droplet can be estimated from the slope of the sections, which indicates that sliding on the liquid membrane moves much faster than crawl mode. c) The magnified details of the droplets (droplets $\mathrm{i}$ and ii) confirm the fast sliding movement of the droplets on the liquid membrane that forms on surface of the seta shaft. Scale bars, a) $100 \mu \mathrm{m}$, and c) $50 \mu \mathrm{m}$.

After the initial stage (before $17.25 \mathrm{~s}$ ), the transport mode of the condensed water droplets completely changes. A thin and continuous liquid membrane is formed on the entire surfaces of the whole seta shaft after the droplets (droplet 1-7) passing (Figure 3a). In Figure 3a (from 18.0221 to 18.0266 s), the water droplet $1^{\prime}$ can fast slide instead of slow crawl on the surface of seta shaft, which is the most significant difference compared with the previous modes. During the sliding process, the volume of the droplet $1^{\prime}$ is much smaller with its radius about $10 \mu \mathrm{m}$ (Figure 3a). More importantly, the average transport velocity $\left(\mathrm{v}^{\prime}\right)$ of droplet $\mathrm{I}^{\prime}$ under sliding mode can be up to $\approx 100 \mathrm{~mm} \mathrm{~s}^{-1}$, which is much faster than the values previously reported. ${ }^{[2,3,5-7,11,12]}$ The detailed process of the sliding transport mode can be seen from the in situ observation of the further enlarged seta (Figure $3 \mathrm{c}$ and Movie S2,
Supporting Information). The seta shaft is wrapped by continuous liquid membrane. This water membrane has the function of fluid lubrication due to its lower friction. Therefore, it can accelerate the movement of the droplets and make the velocity of the droplets that captured from fog increase by several orders of magnitude. The liquid membrane itself can also fast transport the captured fog by means of a sequential liquid layer, which flows on the setae surface. The relatively large droplets (like droplet $\mathrm{i}$ and ii in Figure 3c) can also fast slide with low resistance through the liquid channel between the continuous liquid membrane and the solid surface. After repeated observations over many seta, it is found that, despite the average velocity varies from setae to setae, the two stages of the water transport are observed clearly in all tested samples. 
Undergoing constant evolution, animal hairs hold a variety of specific functions, such as water-repellent hairs on water strider legs, ${ }^{[6]}$ the huge adhesive force generated by gecko foot-hair, ${ }^{[13]}$ and enhanced optical reflection hairs on silver ants. ${ }^{[14]}$ There are also some hairs used as sensors, such as ultrasensitive hairs for fluid, ${ }^{[15]}$ tactile hairs, ${ }^{[16]}$ and chemosensory hairs. ${ }^{[17]}$ Seta, one of the hairs on animal body surface, is also considered for gustatory sensors or mechanosensors. ${ }^{[18]}$ In fact, the special abilities of the setae are all resulted from the unique micro- and nanoscale morphological and structural features of the setae. Here, the gradient linear channels on the conical-shaped setae of desert scorpion in this work should also make contribution to capture water from fog flow and its fast droplet transportation. Therefore, a potential mechanism of this superfast fog capture process has been proposed to demonstrate the water transport mode on the seta (Figure 4). Generally, a water droplet can be driven forward for directional moving by chemical, ${ }^{[19,20]}$ thermal, ${ }^{[21,22]}$ and geometry gradient. ${ }^{[5,8,22-24]}$ The seta with a conical shape can generate a difference of Laplace pressure ${ }^{[5,8,12,23]}$ between the tip and the base (See Theory S1, Supporting Information). On the one hand, the possible driving force for directional water droplet movement arises from the vertebral geometry of the seta shaft, which will generate a difference in Laplace pressure (Figure S5, Supporting Information). On the other hand, the gradient roughness that arises from the gradient channels ${ }^{[5]}$ (gradient width and numbers) on surface of seta generates a surface-energy gradient ${ }^{[5,12,25]}$ (Theory S1, Supporting Information). The forces generated by both the difference of the Laplace pressure $^{[5,23,26]}$ and the gradient surface-energy ${ }^{[19]}$ are the main forces that drive the directional movement of the water droplets on the initial dry surface of seta shaft.

For ease of description, a droplet on the seta of desert scorpion can be divided into boundary layer water (BLW) and upper layer water $(\mathrm{ULW})^{[2]}$ (Figure 4a, Figure S6 and Theory S2, Supporting Information). For the BLW, the main driving forces

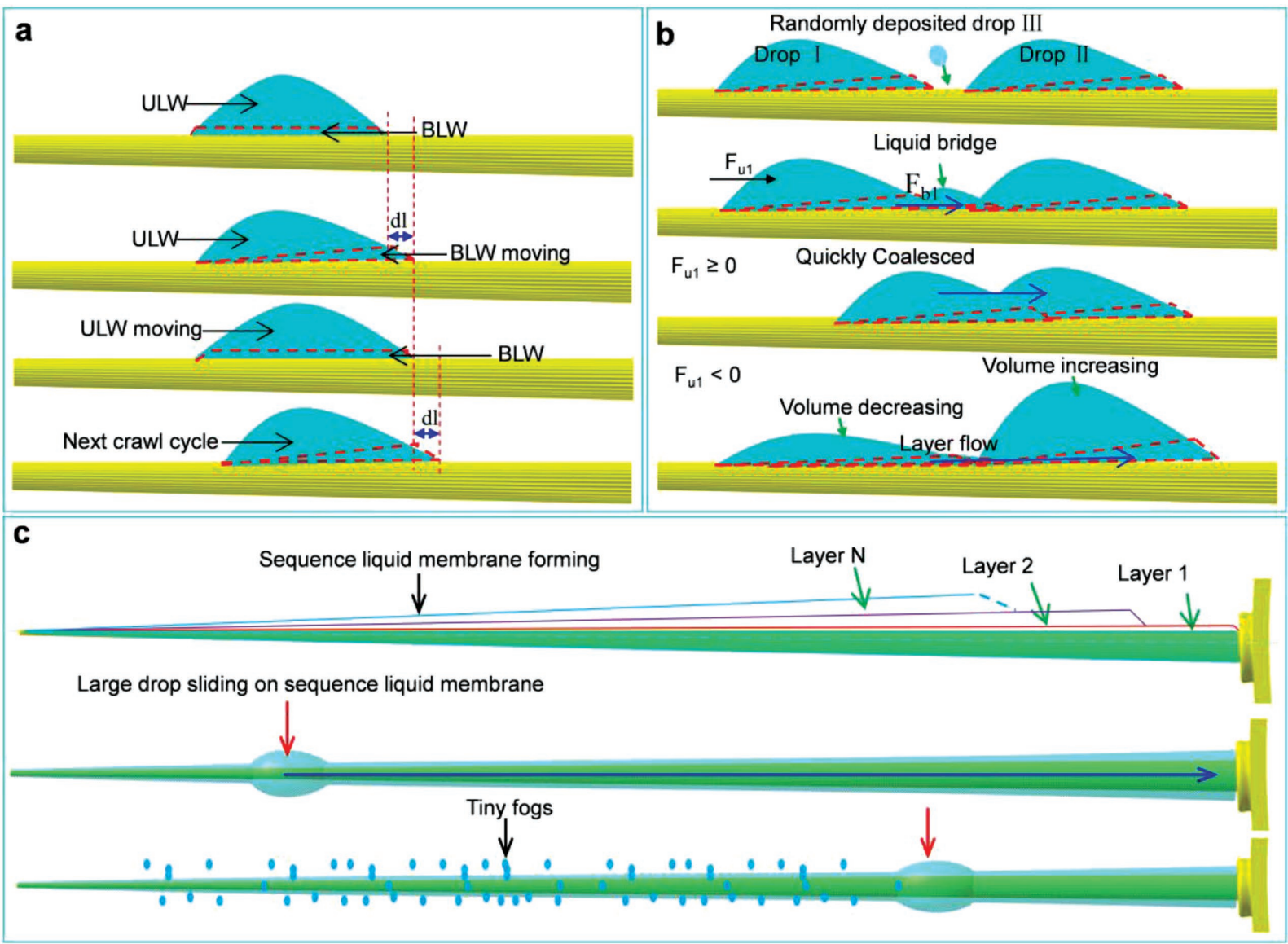

Figure 4. Mechanism of the superfast fog capture strategy of the desert scorpion setae through sliding on the liquid membrane. a) A fog droplet deposited on the seta, BLW is driven forward a distance $(d l)$ by the difference of Laplace pressure and the gradient surface-energy generated by the conical shape with the gradient roughness. As the $d l$ increases, ULW is driven by the BLW and moves forward. The droplet can move in crawl mode from the tip to the base on the seta shaft. b) Droplets I and II deposit on the seta and are separated by its dry part. Then, fog droplet III appropriately deposits on the dry part of seta and connects the BLW of droplet I and droplet II as a liquid bridge. When the net driving force $\left(F_{\mathrm{u}}\right)$ of the ULW, $F_{\mathrm{u}} \geq 0$, the whole droplet I will be quickly driven forward to the droplet II. When $F_{\mathrm{u}}<0$, the BLW will be separated from droplet I and moves to the droplet II. The water droplet transports as a sequence layer flow. The volume of the droplet I decreases, but the volume of the droplet II increases. c) A mount of fog droplets deposited on the seta and wetted the whole surface of the seta. A fast sequence liquid-membrane formed on the seta surface. The BLW is the layer 1 and the main driving layer. The layer 2 to layer $N$ will be driven by the layer 1 . Some big droplets (red arrow) will slide on the liquid membrane formed on the seta surface. 
mostly arise from the difference of Laplace pressure $\left(\Delta P_{\mathrm{b}}\right)$ and the gradient interfacial tension $\left(F_{R}\right)$ of liquid solid. The BLW fills into the microchannels on surface of seta and forms a thin liquid membrane, which composes the main part of the BLW. For the ULW, the main driving forces arise from the difference of Laplace pressure $\left(\Delta P_{\mathrm{u}}\right)$. The net force of $F_{\mathrm{b}}$ and $F_{\mathrm{u}}$ can be expressed as ${ }^{[27]}$ :

$F_{\mathrm{b}}=\Delta P_{\mathrm{b}} A_{\mathrm{b}}+F_{\mathrm{R}}-\gamma_{\mathrm{LG}} L_{\mathrm{LG}}-\gamma_{\mathrm{GS}} L_{\mathrm{GS}}-\gamma_{\mathrm{LL}} L_{\mathrm{LL}}$

$F_{\mathrm{u}}=\Delta P_{\mathrm{u}} A_{\mathrm{u}}+\gamma_{\mathrm{LL}} L_{\mathrm{LL}}-\gamma_{\mathrm{LG}}^{\prime} L_{\mathrm{LG}}$

where, $\gamma_{\mathrm{LG}}$ is the liquid-gas interfacial tension of the BLW, $L_{\mathrm{LG}}$ is the contact line of the liquid-gas, $\gamma_{\mathrm{GS}}$ is the gas-solid interfacial tension of the BLW, $L_{\mathrm{GS}}$ is the contact line of the gas-solid, $A_{\mathrm{u}}$ is the change in cross section area of ULW, $\gamma_{\mathrm{LG}}^{\prime}$ is the liquidgas interfacial tension of the ULW and $\gamma_{\mathrm{LL}}$ is the liquid-liquid interfacial tension between BLW and ULW (see Theory S1 in the Supporting Information), $L_{\mathrm{LL}}$ is the contact line between BLW and ULW.

Initially, the ULW and the BLW are at the same position. They have the same advancing angle $\left(\theta_{\mathrm{A}}\right)$ and receding angle $\left(\theta_{\mathrm{R}}\right)$ for the droplets at the initial time on the seta, where $\gamma_{\mathrm{LG}}=\gamma_{\mathrm{LL}}=0$, $F_{\mathrm{b}}=\Delta P_{\mathrm{b}} A_{\mathrm{b}}+F_{\mathrm{R}}-\gamma_{\mathrm{LG}} L_{\mathrm{LG}}$ and $F_{\mathrm{u}}=-\gamma_{\mathrm{LG}}^{\prime} L_{\mathrm{LG}}$. Then, the BLW moves toward the more hydrophilic side with a length $d l$ by $\Delta P_{\mathrm{b}} A_{\mathrm{b}}+F_{\mathrm{R}}\left(\Delta P_{\mathrm{b}} A_{\mathrm{b}}+F_{\mathrm{R}}>\gamma_{\mathrm{GS}} L_{\mathrm{GS}}\right)$, which arises from the conical shape with gradient roughness of the setae (see Figure S3 in the Supporting Information). The movement of the BLW is unidirectional. ${ }^{[28,29]}$ The BLW fills into the micro channels with the increased length of $d_{1}$. At this time, the contact angle hysteresis ${ }^{[29,30]}$ happens, where $\gamma_{L G}>0$ and $\gamma_{L I}>0$. As the moving length $d l$ of the BLW increases, $\gamma_{\mathrm{LG}}$ and $\gamma_{\mathrm{LL}}$ increase. Therefore, $F_{\mathrm{b}}=0$ and $F_{\mathrm{u}}>0$, the ULW will be fast driven by the BLW to the same position. Then, the BLW keeps moving and wets the dry area of the setae. The next moving cycle continues. All the droplets will keep moving in this "crawl" mode along the setae from the tip to the base. The water transport speed is determined by the BLW wetting speed in this process. Because the wetting speed is low, it limits the water transport speed $(v)$ of all the droplets. The crawl speed of droplet $1\left(v_{1}\right)$ and droplet $1+2$ $\left(v_{2}\right)$ in Figure $3 \mathrm{~b}$ can reflect this characteristics.

As the deposited fog increases, the quantity of the droplets deposited on the seta increases. Some droplets will deposit among the other droplets. As shown in Figure 4b, the droplet I deposits between droplet I and II. The dry area between droplet I and droplet II is quickly wetted by droplet III. The BLW of droplet III connects the BLW of droplet I and II, like a liquid bridge. In this time, the net force of the $\operatorname{BLW}\left(F_{\mathrm{b} 1}\right)$ and the ULW $\left(F_{\mathrm{u} 1}\right)$ of the droplet I can be expressed as:

$F_{\mathrm{b} 1}=\Delta P_{\mathrm{b} 1} A_{\mathrm{b} 1}+F_{\mathrm{R} 1}-1-\gamma_{\mathrm{LL} 1} L_{\mathrm{LL} 1}$

$F_{\mathrm{u} 1}=\Delta P_{\mathrm{u} 1} A_{\mathrm{u} 1}+\gamma_{\mathrm{L} 1} L_{\mathrm{LL} 1}-\gamma_{\mathrm{LG} 1}^{\prime} L_{\mathrm{LG} 1}$

The net force of the BLW $\left(F_{\mathrm{b} 1}\right)$ suddenly increases due to the sudden disappearance of surface tension $\gamma_{\mathrm{LG} 1}+\gamma_{\mathrm{GS} 1}$. When $F_{\mathrm{u} 1} \geq 0$, the BLW will drive the ULW move and get together. For example, in the later period of the crawl stage, the larger droplet $1+2+3$ fast moves toward the large droplet $4+5$ (from 17.17 to $17.19 \mathrm{~s}$ in Figure 3a). The transport speed of droplet $1+2+3\left(v_{3}\right)$ and droplet $1+2+3+4+5\left(v_{4}\right)$ in Figure $3 \mathrm{~b}$ can reflect this characteristics. When $F_{\mathrm{u} 1}<0$, the BLW would separate from the droplet I and move toward the droplet II. The ULW fills on the BLW position and forms new BLW. The BLW keeps moving toward the droplet II. The ULW of the droplet I quickly moves toward the droplet II as this kind of layer mode. The volume of droplet I will decrease, but the volume of droplet II will increase. As shown in Figure 3a (from 18.0221 to 18.0266 s), the droplet 1'moves fast from the tip side to the base side with the volume decreasing during the transport process. The water transport speed of the droplet can be improved to $v^{\prime}$ (Figure 3b).

As the fog deposition increases, the entire surface of the seta shaft would be wetted by the droplets. All the channels of the setae surface are filled with water. A sequence liquid membrane will form from the tip to the base (Figure $4 \mathrm{c}$ and Movie S2, Supporting Information). The liquid membrane can transport the condensed water from the tip to the base without resistance from interfacial tension of liquid-gas and interfacial tension of gas-solid. Some relatively big droplets will be taken by the sequence liquid membrane in the sliding mode. The volume of these droplets will also decrease during the sliding process.

To further demonstrate the role of shape and surface structures on the water transport modes, the artificial setae are successfully fabricated using a biotemplate method (Figure S7, Supporting Information). The original shape and surface structures are both left over on the styreneic block copolymer (SBS) replica (Figure 5a-d, Figures S8 and S9, Supporting Information). In the regard of the wettability of materials or the surface energy, the original chitin and SBS have the similar performance. Besides, according to the mechanism mentioned above, once the materials have the similar shape and surface structures, they should be equipped with the similar fog capture and water transport performance. So, the fog captures and water transport experiments were conducted (Figure 5f). The similar process was observed again (Movie S4, Supporting Information). The shape and the surface structures are thus crucial for the fast sliding water transport in this system and ensure that transport occurs on both the natural and the artificial setae surface much more quickly than those previously reported, ${ }^{[2,3,5-7,11,12]}$ where different wetting modes were established. An unusual strategy of superfast self-support transferring liquid by sliding on the liquid membrane would be superior and expected to replace the traditional strategy of just spreading on interfaces of liquid and solid.

To conclude, we experimentally demonstrate and theoretically analyze a novel strategy for fast transport condensed droplets on a special structured seta of desert scorpion. Our high-speed observation analysis shows that the condensed droplets move in crawl mode when the seta is dry at the beginning. The forces generated by both the difference of the Laplace pressure ${ }^{[5,23,26]}$ (Figure S5, Supporting Information) and the gradient surfaceenergy| ${ }^{[19]}$ (Theory S1, Supporting Information) drive the directional movement of the water droplets on the initial dry surface of seta shaft, forming a liquid membrane continuously wrapped the entire surface of the seta. Due to the loss of interfacial resistance that caused by this hydrodynamically lubricating 

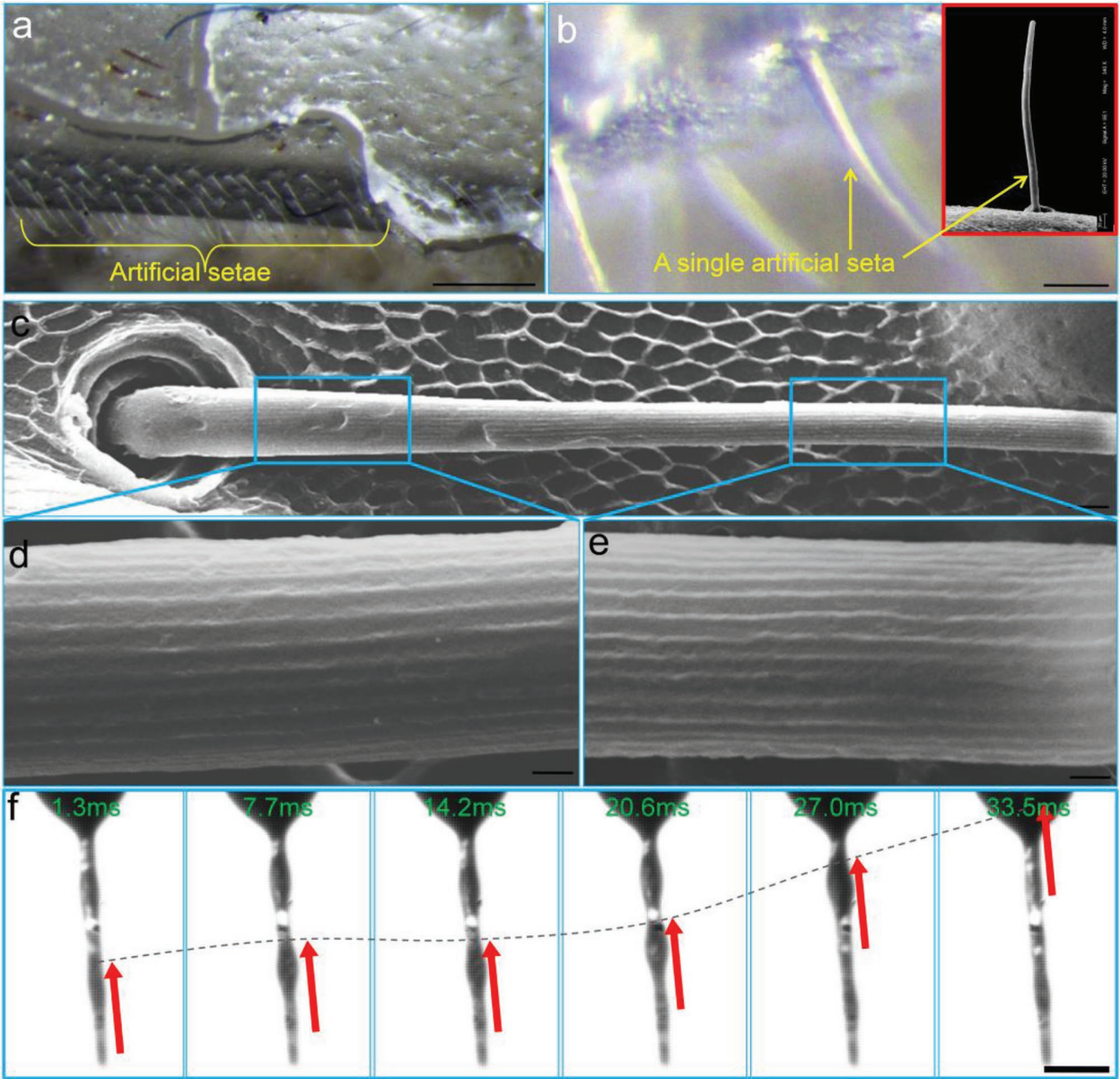

Figure 5. The artificial replica of scorpion seta and its superfast fog capture performance through sliding on the similar liquid membrane. Panels (a) and (b) are the optical microscope images of the bio-replica of the scorpion seta. The artificial replica is transparent to distinguish between scorpion seta. Panels (c-e) are the SEM images of a single artificial replica seta. The artificial replica replicates the microcannels of the seta. f) The fog capture process of the artificial replica indicated that the superfast fog capture through sliding on the similar liquid membrane can be achieved in artificial materials using the same mechanism. Scale bars, a) $200 \mu \mathrm{m}$, b) $20 \mu \mathrm{m}$, c) $10 \mu \mathrm{m}, \mathrm{d}$,e) $2 \mu \mathrm{m}$, and f) $20 \mu \mathrm{m}$.

water membrane, the movement of droplets could be significantly accelerated. Then, the velocity of the droplets that captured from fog can accordingly increase several orders of magnitude. This cannot be achieved on a nonwettable surface with nonzero contact angle. We regard this study as the primary step in what we hope there will be an ongoing series of technological developments. The next step will be to test a variety of liquids and to define those design parameters that will allow us to capture fog of different liquids. To achieve a proper fog capture ability of the conical setae with linear gradient channels, it will be important to take into account of the combined effects of surface properties of the structure and the features of different liquid (surface tension, molar volume, and molecular shape and size of the fog). By optimizing the distribution, surface structures and morphologies of the setae, almost perfect fog capture surfaces could be engineered for omnidirectional fog capture (Figure S4, Supporting Information) in thin fog conditions (Movie S3, Supporting Information). Such fog capture surfaces and the sliding movement in liquid membrane on scorpion seta could be adapted to design novel materials and movable devices for fast and efficiently fog collector. ${ }^{[31]}$ If regulated properly, this bionic control of flows are also of significant value in several industrial applications, such as the design and operation of microfluidic and integrated DNA analysis devices.

\section{Experimental Section}

Biological Prototype: The living scorpions (P. transvaalicus) were purchased from School of Life Science, Northeast Normal University, Changchun, China. After identification, it was confirmed that they were native to the dry desert of South Africa. The pincers, legs, and tail of the desert scorpion were all cut down from its body after it was fainted by ether. The single seta was carefully selected and fixed on the sample frame.

Characterizations of the Microstructures: The optical images of the setae on the desert scorpion were recorded by a digital camera (Canon 7D). The setae distributed on the pincers and other parts of this desert scorpion were obtained by a stereomicroscope (Olympus SZ-61). The detailed microstructures of different position of the seta shaft and the socket were observed by a field-emission scanning electron microscope (ZEISS, $\sum$ ICMA 500, Germany) at an accelerating voltage of $\approx 3.0 \mathrm{KV}$.

Fog Capture Experiments and Observation: The seta was located on the scorpion and investigated under a saturated fog flow with a velocity 
of $\approx 20-30 \mathrm{~cm} \mathrm{~s}^{-1}$ (by an ultrasonic humidifier using Milli-Q water). The behavior of the water droplets was recorded by a high-speed camera system (PCO. dimax HD, Germany) with a microscopic magnification lens (Navitar 1-62922, Navitar 1-60112 1.5×Lens, American). The scorpion was carefully fixed on the retort stand by a ring clamp. Three different setae with different angles $\left(0^{\circ}, 45^{\circ}\right.$, and $\left.90^{\circ}\right)$ with respect to the direction of gravity were chosen for the experiments. The fog flow was jetting from a $10 \mathrm{~cm}$ diameter pipe at room temperature $\left(\approx 23{ }^{\circ} \mathrm{C}\right)$. The droplet speed was analyzed by video motion analysis software (TEMA MOTION, Sweden).

\section{Supporting Information}

Supporting Information is available from the Wiley Online Library or from the author.

\section{Acknowledgements}

This work was supported by the National Natural Science Foundation of China (Nos. 51325501, 51505183, and 51290292), China Postdoctoral Science Foundation Funded Project (No. 2015M571360), Joint Construction Project of Jilin University and jilin Province (No. SF2017-3-4), Program for JLU Science and Technology Innovative Research Team, and Outstanding Young Talent Fund of Jilin Province (No. 20170520095JH).

\section{Conflict of Interest}

The authors declare no conflict of interest.

\section{Keywords}

bioinspired materials, droplet transportation, gradient channel structures, scorpion setae, sliding movement

Received: May 28, 2018

Revised: July 16, 2018

Published online: August 12, 2018

[1] Z. W. Han, Z. Z. Mu, B. Li, Z. Wang, J. Q. Zhang, S. C. Niu, L. Q. Ren, ACS Nano 2016, 10, 8591.

[2] H. W. Chen, P. F. Zhang, L. W. Zhang, H. L. L. Iu, Y. Jiang, D. Y. Zhang, Z. W. Han, L. Jiang, Nature 2016, 532, 85.

[3] Z. Pan, W. G. Pitt, Y. M. Zhang, N. Wu, Y. Tao, T. T. Truscott, Nat. Plants 2016, 2, 16076.

[4] L. Guo, G. H. Tang, Int. J. Heat Mass Transfer 2015, 84, 198.

[5] J. Ju, H. Bai, Y. M. Zheng, T. Y. Zhao, R. C. Fang, L. Jiang, Nat. Commun. 2012, 3, 1247.

[6] X. F. Gao, L. Jiang, Nature 2004, 432, 36.
[7] A. R. Parker, C. R. Lawrence, Nature 2001, 414, 33.

[8] M. J. Liu, S. T. Wang, L. Jiang, Nat. Rev. Mater. 2017, 2, 17036.

[9] C. C. Liu, Y. Xue, Y. Chen, Y. M. Zheng, Sci. Rep. 2015, 5, 17757.

[10] L. Zhai, M. C. Berg, F. C. Cebeci, Y. Kim, J. M. Milwid, M. F. Rubner, R. E. Cohen, Nano Lett. 2006, 6, 1213.

[11] K. C. Park, P. Kim, A. Grinthal, N. He, D. Fox, J. C. Weaver, J. Aizenberg, Nature 2016, 531, 78.

[12] Y. M. Zheng, H. Bai, Z. B. Huang, X. L. Tian, F. Q. Nie, Y. Zhao, J. Zhai, L. Jiang, Nature 2010, 463, 640.

[13] K. Autumn, Y. A. Liang, S. T. Hsieh, W. Zesch, W. P. Chan, T. W. Kenny, R. Fearing, R. J. Full, Nature 2000, 405, 681.

[14] N. N. Shi, C. C. Tsai, F. Camino, G. D. Bernard, N. F. Yu, R. Wehner, Science 2015, 349, 298.

[15] P. Fratzl, F. G. Barth, Nature 2009, 462, 442.

[16] F. G. Barth, S. S. Nemeth, O. C. Friedrich, J. Comp. Physiol., A. 2004, 190, 523.

[17] S. R. Shanbhag, D. P. Smith, R. A. Steinbrecht, Arthropod Struct. Dev. 2005, 34, 153.

[18] a) S. R. Shanbhag, B. Muller, R. A. Steinbrecht, Int. J. Insect Morphol. Embryol. 1999, 28, 377; b) S. A. Ochieng, K. C. Park, J. W. Zhu, T. C. Baker, Arthropod Struct. Dev. 2000, 29, 231; c) K. Jorgensen, T. J. Almaas, F. Marion-Poll, H. Mustaparta, Chem. Senses 2007, 32, 863.

[19] a) S. Daniel, M. K. Chaudhury, J. C. Chen, Science 2001, 291, 633; b) M. K. W. Chaudhury, G. M. Whitesides, Science 2010, 256, 1539.

[20] S. C. Hernandez, C. J. C. Bennett, C. E. Junkermeier, S. D. Tsoi, F. J. Bezares, R. Stine, J. T. Robinson, E. H. Lock, D. R. Boris, B. D. Pate, J. D. Caldwell, T. L. Reinecke, P. E. Sheehan, S. G. Walton, ACS Nano 2013, 7, 4746.

[21] a) A. L. Yarin, W. X. Liu, D. H. Reneker, J. Appl. Phys. 2002, 91, 4751; b) S. Mettu, M. K. Chaudhury, Langmuir 2008, 24, 10833; c) Q. W. Dai, M. M. Khonsari, C. Shen, W. Huang, X. L. Wang, Langmuir 2016, 32, 7485.

[22] Q. W. Dai, W. Huang, X. L. Wang, Langmuir 2015, 31, 10154

[23] E. Lorenceau, D. Quere, J. Fluid Mech. 2004, 510, 29.

[24] J. L. Zhang, Y. C. Han, Langmuir 2007, 23, 6136.

[25] a) J. T. Yang, Z. H. Yang, C. Y. Chen, D. J. Yao, Langmuir 2008, 24, 9889; b) C. Sun, X. W. Zhao, Y. H. Han, Z. Z. Gu, Thin Solid Films 2008, 516, 4059; c) G. P. Fang, W. Li, X. F. Wang, G. J. Qiao, Langmuir 2008, 24, 11651.

[26] a) P. Renvoise, J. W. M. Bush, M. Prakash, D. Quere, EPL 2009, 86, 39901; b) H. Bai, X. L. Tian, Y. M. Zheng, J. Ju, Y. Zhao, L. Jiang, Adv. Mater. 2010, 22, 5521.

[27] J. S. Rowlinson, B. Widom, Molecular Theory of Capillarity, Clarendon Press, Oxford, UK 1982.

[28] a) K. H. Chu, R. Xiao, E. N. Wang, Nat. Mater. 2010, 9, 413; b) N. A. Malvadkar, M. J. Hancock, K. Sekeroglu, W. J. Dressick, M. C. Demirel, Nat. Mater. 2010, 9, 1023.

[29] Y. Chen, B. He, J. H. Lee, N. A. Patankar, J. Colloid Interface Sci. 2005, 281, 458.

[30] M. Morita, T. Koga, H. Otsuka, A. Takahara, Langmuir 2005, 21, 911.

[31] a) M. J. Estrela, J. A. Valiente, D. Corell, M. M. Millan, Atmos. Res. 2008, 87, 324; b) M. Rajaram, X. Heng, M. Oza, C. Luo, Colloids Surf., A 2016, 508, 218; c) M. J. Estrela, J. A. Valiente, D. Corell, D. Fuentes, A. Valdecantos, Agric. For. Meteorol. 2009, 149, 1896. 